\title{
ESTUDIO DE LA MORTALIDAD MATERNA EN CUBA
}

Prof. Armando Peralta Quintero*

Prof. Pedro Valdés Vivó**

Prof. Eduardo Cutié León***

La Tasa de la Mortalidad Materna constituye uno de los indicadores más importantes para el análisis no solo del funcionamiento de nuestras unidades hospitalarias sino también como una unidad de medida del trabajo realizado en toda la organización obstétrica del País.

Este estudio comprende la totalidad de las Muertes Maternas ocurridas en el País durante el período de 1959 a 1969.

A los efectos de este estudio incluímos como Mortalidad Materna, la debida a causas que tienen relación directa con el embarazo, parto y puerperio, las cuales incluyen los números 630 a 678 de la VIII Clasificación Internacional de Enfermedades (640 a 689 de la VII). Incluímos en nuestra clasificación las muertes ocurridas por embarazo ectópico y mola hedatiforme. Las tasas de mortalidad de 1959 a 1965 se mantienen por encima de $10 \times 10.000$ excepto $1961 \mathrm{con}$ 9,4 (Tabla № 1).

Se observa una disminución a partir de 1964 de 11,3 a 7,6 en 1969.

Las agrupamos en cinco causas: ( Tablas Nos. 2 y 3 ).

A) Aborto.
B) Hemorragia.

C) Infecciones.

D) Toxemia.

E) Otras causas.

TABLA N: 1

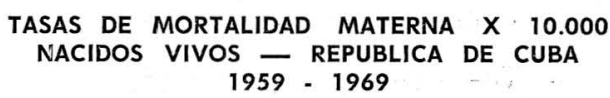

\begin{tabular}{lc}
\hline Años & Tasa $\times 10.000$ N. V. \\
\hline 1959 & 11,5 \\
1960 & 11,6 \\
1961 & 9,4 \\
1962 & 10,7 \\
1963 & 11,2 \\
$1964{ }^{*}$ & 11,3 \\
$1966\left(^{*}\right)$ & 11,3 \\
$\left.1967{ }^{*}\right)$ & 8,1 \\
$1968\left(^{*}\right)$ & 9,4 \\
\hline
\end{tabular}

NOTA: (*) Datos Provisionales.

FUENTE: Departamento de Estadísticas, Ministerio de Salud Pública.

* Departamento de Obstetricia y Ginecología de la Escuela de Medicina de la Universidad de La Habana.

** Departamento de Obstetricia y Ginecología de la Escuela de Medicina de la Universidad de La Habana.

*** Departamento de Obstetricia y Ginecología de la Escuela de Medicina de la Universidad de La Habana. Secretario de la Sociedad Cubana de Obstetricia y Ginecología. 
Desglosada por rubros vemos que la tasa de mortalidad por Hemorragia ha descendido de 3,4 (67 casos) en 1959 a 1,7 ( 45 casos) en 1964, a 2,9 (67 casos) en 1968.

Las de Infecciones de 1,0 (20 casos) en 1959 a 0,9 ( 24 casos) en 1964 y a 1,3 (31 casos) en 1968.

La de Toxemia de 2,2 en 1959 (43 casos) a 1,7 (45 casos) en 1964 y a 0,8 (18 casos) en 1968.

La tasa de mortalidad por Aborto aumenta de 1,2 en 1959 ( 25 casos) a 3,1 en 1964 ( 84 casos) y a 2,5 (59 casos) en 1968.

La mortalidad por Aborto aumenta de 1959 a 1965 para descender en 1968.

Un subregistro en los primeros años explica en parte este aumento.

Este rubro ocupa la Primera Causa de Muerte de nuestro País seguida de Hemorragia, Infección, Muerte por Otras Causas y por último Toxemia.

Este esquema corresponde al análisis por causas de cualquier país subdesarrollado ya que en los países con alto desarrollo los rubros Hemorragia e Infecciones ocupan tasas muy bajas siempre después de la Toxemia y Muerte por Otras Causas que están en los primeros lugares.

Analizando las causas de muerte por provincias durante el año 1968 vemos como las provincias orientales (principalmente Oriente Sur) mantienen las tasas más altas. Principalmente los rubros correspondientes a Toxemia, Infecciones y Hemorragia.

Las características geográficas, la distribución de la población así como el más bajo por ciento de parto institucional son factores que explican 
TABLA N: 3

MORTALIDAD MATERNA SEGUN CAUSAS - AÑOS 1959 A 1965 - CUBA

\begin{tabular}{ccccccc}
\hline Año & Infecciones & Toxemia & Hemorragia & Aborto & Otras causas & Total \\
\hline 1959 & 1,0 & 2,2 & 3,4 & 1,2 & 3,4 & 11,5 \\
1960 & 0,8 & 3,4 & 3,1 & 1,3 & 2,8 & 11,6 \\
1961 & 0,8 & 2,4 & 2,0 & 1,3 & 2,7 & 9,4 \\
1962 & 0,6 & 2,6 & 2,5 & 1,9 & 2,9 & 10,7 \\
1963 & 0,9 & 2,2 & 2,2 & 2,3 & 3,3 & 11,2 \\
1964 & 0,9 & 1,7 & 1,7 & 3,1 & 3,8 & 11,3 \\
1965 & 0,8 & 1,8 & 2,0 & 3,9 & 2,8 & 11,3 \\
\hline
\end{tabular}

NOTA: Tasas por 10.000 Nacidos Vivos.

estas diferencias de tasas con las provincias occidentales.

Como factores capaces de explicar el descenso de nuestra mortalidad podemos citar:

1. La creación de una Comisión Nacional para la Discusión de las Muertes Maternas.

En 1963 es creada por el Vice-Ministerio de Asistencia Médica una Comisión Nacional para el estudio y discusión de todas las muertes maternas.

Esta Comisión, constituída por profesores del Departamento de Obstetricia y Ginecología de la Escuela de Medicina de la Universidad de La Habana analizaba las muertes ocurridas en el País a través de las Historias Clínicas que eran enviadas al Ministerio de Salud Pública, discutiendo aquellas con fallas o deficiencias médicas $u$ organizativas.

Posteriormente en Febrero de 1968 y en virtud de una Resolución Ministerial esta Comisión es modificada en su estructura y metodología de trabajo.

Se crea una Comisión Nacional constituída por Profesores de la Escuela de Medicina y Miembros del
Grupo de Gineco-Obstetricia del Ministerio de Salud Pública que radica en el Nivel Central del Ministerio de Salud Pública, en La Habana.

La Comisión Nacional tiene facultad para discutir o rediscutir cualquier muerte materna ocurrida en el País.

Son creadas 8 Comisiones Provinciales (una en cada Provincial) constituída por 3 a 5 especialistas (profesores donde tenemos Universidades) que analizan y discuten la totalidad de las muertes de sus respectivas provincias.

Al fallecimiento de toda gestante parturienta o puérpera hasta los 90 días posteriores al parto, el Director de la Unidad Hospitalaria donde ocurrió la muerte en las primeras 48 horas envía original y una copia de la Historia Clínica a la Comisión Provincial, archivando una copia en la Unidad.

El fallecimiento es comunicado previamente por telegrama a la Comisión Provincial.

La Comisión designa un ponente de uno de sus miembros, el cual en los 45 días siguientes a la muerte procede a la discusión de la Historia Clínica en la unidad donde ocurrió la 
muerte materna con la presencia de la totalidad del personal facultativo del Servicio de Gineco-Obstetricia.

El carácter educativo que tiene esta discusión es uno de los fines esenciales de la misma. Los elementos aportados por el análisis son recogidos como sugerencias para superar deficiencias que se hubieran puesto de manifiesto $y$ en esto es concreta la responsabilidad de la Comisión.

Se precisa la causa de la muerte basándose en el resultado de la necropsia.

Las conclusiones de la Comisión Provincial son recogidas en un acta por triplicado —una es enviada a la Comisión Nacional, otra al archivo de la Comisión Provincial y la otra queda en la Unidad-.

Los reparos de los certificados de defunción ( $\mathrm{si}$ fuere necesario) son realizados al concluir la discusión y enviados al Departamento de Estadísticas Provincial el cual realiza las correcciones necesarias al certificado de defunción expedido en el momento del fallecimiento, para su más correcta codificación.

Reportes mensuales, semestrales y anuales se realizan por estas Comisiones enviando copias al Nivel $\mathrm{Na}$ cional.

2. El aumento del parto institucional de un $64 \%$ en 1963 a $91,4 \%$ en 1968.

$\begin{array}{ll}1963 & 64 \% \\ 1964 & 67 \% \\ 1965 & 73,6 \% \\ 1966 & 80 \% \\ 1967 & 86,4 \% \\ 1968 & 91,4 \%\end{array}$

3. El aumento del número de camas Gineco-Obstétricas.
Año 1968

Total de camas Gineco-Obstéricas

Total de camas Obstétricas

Total de camas en hospitales de Maternidad Indices:

Obstetricia

Gineco-Obstetricia

$0,5 \times 1.000$ habitantes $0,7 \times 1.000$ habitantes

\section{La creación de los Hogares Ma-} ternos.

Instituciones cuyo objetivo fundamental es el de acercar a los centros hospitalarios a las gestantes que viven distantes de los mismos y donde las características geográficas dificultan el transporte.

$N^{0}$ de hogares maternos: Antes de 19590 $1969 \quad 17$

Estos Hogares Maternos están ubicados en las provincias donde el por ciento de parto institucional es más bajo y en donde los ingresos geográficos ocupan camas que pueden ser utilizadas en el ingreso de pacientes con alto riesgo obstétrico.

5. Una mejor cobertura obstétrica.

1) Aumento del número de especialistas y médicos generales dedicados a la especialidad.

\section{Especialistas Graduados}

Tenemos actualmente en planes de Residencia:

Residentes

Internos

Médicos que han terminado su Internado en la especialidad, ubicados en zonas rurales durante dos años y que posteriormente regresan a completar su especialidad:

Post-graduados ler. año
Post-graduados $2^{0}$ año

Total 
2) Formación e incremento de la Enfermería y Enfermería Obstétrica.

De 6 escuelas en 1959 a 17 escuelas de Enfermería General.

Personal de Enfermería graduado hasta 1968:

Enfermeras Generales

Auxiliares de Enfermería

Enfermeras Obstétricas

Ubicación del personal graduado en las áreas rurales. Utilizando parte de sus horas de trabajo en la captación de gestantes que no concurren a la consulta pre-natal.

\section{Incremento de la Atención pre- natal.}

El número de controles pre-natales ha ido incrementándose en los últimos años.

En 1968 el número de consultas por parto fue de 6 .

7. Creación de Bancos de Sangre. en Provincias y Regionales.

En 1959, 1. En 1969, 17.

Es de todos conocido la importancia de la Hemoterapia en el tratamiento de las hemorragias del tercer trimestre de la gestación y de las del post-parto inmediato.

Además cada hospital cuenta con Servicio de Transfusiones.

8. La Normación con carácter nacional de la especialidad, tanto en Obstetricia como en Ginecología.
Estas normaciones en las cuales han participado la totalidad de los especialistas en Gineco-Obstetricia tienen como objetivo principal la unificación de criterios diagnósticos y terapéuticos fundamentalmente en la atención pre-natal y las patologías gineco-obstétricas más importantes, incluyendo la atención del recién nacido normal y patológico.

(Todos nuestros especialistas, residentes e internos las conocen y aplican ).

La supervisión del cumplimiento de las Normas, principalmente la atención pre-natal, es trabajo jerarquizado en las funciones de los Jefes de Servicio y Funcionarios de Salud Pública.

9. La Educación Sanitaria que se realiza utilizando masivamente los medios radiales, televisados y de prensa del País, haciendo hincapié en la atención pre-natal.

10. Leyes de Protección a la embarazada.

Toda gestante tiene derecho a disfrutar de una licencia con salario completo hasta 250.00 pesos, durante 12 semanas. Tiene, además, la disminución de una hora al día en su jornada de trabajo diaria durante un año para la atención y lactancia del recién nacido.

Estas leyes contemplan, también, una licencia sin sueldo durante un año si la madre lo solicita. 